\title{
THE PROBLEM OF ANTHTROPOCENTRIC SEMANTICS OF A POLYSEMANTIC WORD
}

\author{
(C) L. G. Yusupova \\ Ural State Mining University \\ 30 Kuibyshev Street, 620144 Yekaterinburg, Russia. \\ Phone: +7 (343) 2514089. \\ Email:lyalyax@bk.ru
}

\begin{abstract}
This article is supposed to provide the factual material demonstrating that all the meanings of a polysemantic word represent the interaction of two mental spaces, namely a systematic meaning and a lexical invariant. It considers anthropocentric semantics to be one of the main trends of linguistic anthropology, which constructs a model of a human by the language means. The author of the article gives detailed consideration to the basic method of determining a lexical invariant by means of a cognitive analysis of lexical meanings through the reconstruction of the semiosis processes, an empirical semantic analysis, and the analysis of dictionary definitions. Since a nominative non-derivative meaning is an integral part of a lexical invariant and the basis for the understanding of all the figurative meanings, it is formulated on the basis of the dictionary definitions using a componential analysis with a frequency principle.
\end{abstract}

Keywords: cognitive analysis of lexical meanings, anthropology, anthropocentric semantics, nominative non-derivative meaning, lexical invariant.

Anthropocentrism is a person's ability to perceive surrounding reality, breaking it through the prism of his consciousness and creating his own picture of the world, part of which he realizes himself, and which he reproduces in speech-thinking activity.

The basis of the anthropocentric construction of a picture of the world is the peculiarity of the reflection of reality by the human consciousness, namely, going beyond the limits of direct sensory experience into the sphere of abstract rational experience, that is, a reflection of reality in its complex causal connections and relationships [2].

The anthropocentric paradigm has deep historical roots in science. One of the first scientists who expressed the idea of creating a science of language on an anthropological basis is the eminent German scientist V. von Humboldt. According to his opinion, "learning a language does not exclude the ultimate goal, and together with all other areas it serves the highest and common goal of the joint aspirations of the human spirit, the goal of the human spirit knowing itself and its attitude to everything that is visible and hidden around" [3, p. 234].

The ideas put forward by V. von Humboldt are reflected in the scientific research of A. A. Potebnya. Contemplating on the development of language, he, following Humboldt, asserts its anthropocentric character, "In reality, language develops only in society, and not only because a person is always a part of the whole to which he belongs, namely his tribe, people, humanity, not only because of the need for mutual understanding as a condition of the possibility of public enterprises, but also because a person understands himself only when he has experienced the clarity of his words" [1, p. 87].

To a large extent an anthropological thought determined the emergence of anthropology as an independent field of knowledge. Descartes's principle "I think, therefore I am" represented the importance of the subjective personality formation. According to R. Descartes, the basis for the understanding of the culture was self-consciousness, which was not self-sufficient yet and did not get complete independence due to the fact that the ultimate authority was connected with God as an infinity, an omnipotent being who set a man in the right way. Thus, a person's awareness of himself as a subject meant his final alienation from the cosmos that had given birth to him. Descartes laid the foundations of a new European tradition to consider selfconsciousness as the basis of the culture resistant to nature. The recognition of the sovereignty of a thinking subject seeking support only within himself can be called a turn to anthropology.

In the cognitive scientists' opinion, meanings of the words originate and are formed not only and not so much at the level of semantics as in some conceptual formations and schemes related to the cognitive system. This article aims at establishing basic cognitive mechanisms underlying the formation of polysemy of a word and the definition of a lexical invariant (LI). Factual material of the article is supposed to demonstrate that all the meanings of a polysemantic word represent the interaction of two mental spaces namely a systematic meaning and a lexical invariant. The problem of the semantic unity of a polysemantic word can be solved by the recognition of an out-of-context invariant meaning of a generalized character being formed on the basis of the functioning of a word at the level of the language system [4].

An invariant meaning is formed by a person in the course of time when secondary meanings are actualized in different contexts. When we talk about variation, the question of the invariant of all the variants is certain to arise. For example, a child in the process of his development starts thinking why we use a Russian word ручка, speaking affectionately about a hand ("he kissed her lovely hand"), a door handle, a suitcase handle, a 
kettle bail, or a pen as a writing implement (a ballpoint pen). While doing it, we generalize the previous experience of using these meanings in the context, and by means of abstraction we come to the conclusion that ручка is something related to a hand as well as something held or taken by a hand. Therefore, it is possible that an abstract semantic kernel resulting from numerous actualizations of figurative meanings of a given polysemantic word is formed in consciousness on the basis of generalized semantic components.

The basic method used to determine a lexical invariant $(\boldsymbol{L I})$ is a cognitive analysis of lexical meanings through the reconstruction of the semiosis processes, empirical semantic analysis, and the analysis of dictionary definitions [7, 8].

Since a nominative non-derivative meaning (NNM) is an integral part of a LI and the basis for the understanding of all the figurative meanings, it is formulated on the basis of dictionary definitions by means of componential analysis with a frequency principle. This meaning includes necessary and sufficient components for the word to be recognized at the level of ordinary consciousness. NNM is essential to the understanding of a metaphor, and the degree of its understanding and interpretation depends primarily on the comprehension of a literary meaning and its interaction with a context. On the other hand, it is important to identify the abstract semantic kernel and its role in the semiosis processes and the development of a semantic structure of a polysemantic word [8].

The NNM of the words under analysis is formulated on the basis of 8-10 definitions from 25 dictionaries. The choice of dictionaries depends on the word being analyzed.

The initial part of the analysis aims at formulating a nominative non-derivative meaning based on the most frequent semantic components. Some of the presented definitions are of a scientific character and contain the least number of frequency characteristics necessary and sufficient to identify a corresponding concept at the level of ordinary consciousness [5].

The generalized nominative non-derivative meaning of the noun arm can be the upper limb of the human body extending from the shoulder to the wrist. The next step to define a LI is to interpret the meanings of the word on the basis of the determined NN meaning.

Since lexical-semantic variation (LSV) (1) conveys a generalized concept of a person's arm, meaning (2) (of the word arm), each of the forelimbs of an animal ("The monkey was required to move its arm within a horizontal plane" "I looked down and saw it there, a fearsome looking thorn dug deep into the flesh of the poor animal's arm") [ODE], is narrower than the first one because it only relates to a limb of an animal. This meaning is based on the defined NN meaning. The same applies to LSV (3), a flexible limb of an invertebrate animal, e.g., an octopus.

Arm (4) is a sleeve of a garment (The shirt has the same pattern as the short sleeve shirts except for an additional pattern over the biceps and the elbow on both arms). It is a metonymy because part of the body is used to mean a piece of clothing closely associated with it, namely the word arm refers to a sleeve put on it.

The word under analysis has a large number of metonymic meanings. In this way, arm (5), an ability to throw a ball skillfully (Davis has one of the best arms in the organization, but his repertoire remains a work in progress, is a metonymy for being skillful at performing actions associated with a part of a human body. It should be emphasized that the arm throwing a ball in a sports game is called an arm when the ball is thrown masterly.

Arm (6), an athlete with an ability to throw a ball skillfully (He wasn't the best arm in the outfield, but his performance at the plate more than compensated.), is, in our opinion, a second-stage metonymy resulting from a complex transfer of the meaning. At first, an arm is a metonymy for the ability to throw a ball skillfully because it is associated with this part of a human body, and then according to the model "part of the whole" an arm refers to the athlete skillful at throwing a ball.

Similarly to the word hand "helper", the next meaning is a metonymy based on the model when a part stands in for the whole (synecdoche). Arm (7) is used to refer to the holding of a person's arm in support or companionship (Ever the gentleman he offered her arm for support and she accepted thankfully) $[\mathrm{ODE}]$. Here the arm to be leant on refers to the person who helps.

Although the next meaning is similar to the previous one (synecdoche), the Oxford dictionary distinguishes it from the others. Arm (8) is used to refer to something perceived as powerful and protective (Priss kept a protective arm around her lover's waist, not too tight, not too loose.) [ODE] (synonyms: reach, power, force, authority, strength, might, potency). In this meaning the word arm is often used with an adjective protective.

Arm (9) is something, such as a support on a chair, that is designed to cover or support the human arm [AHDEL]; a side part of a chair or other seat on which a sitter's arm can rest (She got down on her knees, poked her head over the arm of a chair and stared at us as if she had been electrocuted.) [ODE]. It can be interpreted as a metonymy based on the association by contiguity because part of the chair specifically designed for a human arm begins to be referred to as an arm. This LSV can also be analyzed as a metaphor, because the semantic components underlying the word arm as an armrest imply a comparison based on the identifying and functional features associated with the projection of an armchair, long, horizontal, used to support an arm of a seated person.

Another arm (10), one of the types of troops of which an army is composed, such as infantry or artillery (The artillery arm has produced many great generals, most notably Napoleon) [ODE], is an excep- 
tional case for a componential analysis. This meaning can be interpreted as a metaphor if it is assumed to be based on the comparison of the type of troops such as artillery or infantry with human arm (1) as the upper limb of the human body extending from the shoulder to the wrist. Artillery or infantry is part of a larger military unit (army) to the same extent as an arm is a mere part of the entire human body. In this case, it is possible to distinguish the following semantic components: one of the types of troops, of which the army is composed (as units of soldiers who fight on foot with small arms). The same meaning can be interpreted as metonymy if we assume that arms stand in for people who perform activities using these arms (in this case they fight with weapons (arms) in their hands (arms).

The metaphors presented below reflect their comparison with the appearance and function of a human arm. This part of the study aims at demonstrating what acts as a starting basis in the formation and decoding of the metaphorical meanings. At the same time, the analysis is based on the principles of anthropometry, thus relying on the corresponding images of perception.

So, metaphor arm (11), a narrow strip of water or land projecting from a larger body (Shuswap Lake is shaped like an $H$ and is made up of the four large arms: the Shuswap Lake Main Arm, Salmon Arm, Anstey Arm, and Seymour Arm.) (synonyms: inlet, creek, cove, fjord, bay, voe) [ODE], is based on the comparison of a narrow strip of water or land with a human arm. A narrow strait, a shoal, or a sandbar in a river or sea is a long and narrow landform projecting from a larger body of land or water to the same extent as an arm is an extension (projection) from a human body. This metaphor is interpreted on the basis of identifying semantic components: strip of water/land, projecting from a larger part, narrow.

Arm (12) is a branch or division of a company or organization (First, it is given direction by a political arm, or college, of Commissioners, but the college is unelected) [ODE] (synonyms: branch, section, department, division, subdivision, wing, sector, chapter, lodge, detachment, agency, office, bureau, offshoot, satellite, extension). In this case, the metaphorical meaning "subsidiary company/organization" is based on the comparison of a branch of a larger organization with a person's arm. In the same way as a branch is a "daughter" of the company or enterprise, a human arm is a branch/projection of the entire human body. Here it is necessary to distinguish such components as a division of a company/organization, smaller than the main company/organization.

Arm (13) is math. each of the lines enclosing an angle (One of the arms of angle [alpha] and one of the arms of angle [beta] are extended by the same amount) [ODE]. This metaphorical meaning is based on the image of two rays passing through one point and forming the sides of the corner and associated with the hands projecting from a human body. The arms spreading by a person may well resemble an angle in the form of a geometric figure. In this LSV, it is possible to dis- tinguish such components as combination of two halflines/rays, with a common endpoint. Besides, the semantics of the meanings half-line and ray potentially implies components long and thin, which are likely to mean an arm.

Arm (14) is the part of an anchor from the crown to the fluke [Meriam]. This LSV is based on the comparison of the part of the anchor from its "crown to the fluke" with arm (1) in appearance. Accordingly, the crown and the fluke of an anchor resemble the arms spread by a man. This metaphor has such components as the part of an anchor, long, narrow.

Arm (15) is any of the usually two parts of a chromosome lateral to the centromere [Meriam]. In this case, the metaphor is based on the comparison of two lateral parts of a chromosome with human arms. The meaning is interpreted by means of the following identifying components: one of the two threadlike structures of nucleic acids, carrying genetic information, lateral.

Arm (15) is a thing comparable to an arm in a form or function; anything considered to resemble an arm in appearance, position, or function [MD]. The illustrations provided by the dictionaries are only a part of the possible nominations resulting from a group of invariant components of the word: arm of a counterweight/completed machine/cables/stabilizer/cultivators/bridge/tractor/robotic/landing/sea/record player etc. As can be seen from the examples, there can be actually an unlimited number of references, and the word itself becomes polysemantic. This actually means that the semantic core underlying all the meanings of the word arm will have the most generalized abstract character.

Then, it would be reasonable to illustrate how the word arm is used in phraseological units (FU). All of them have anthropocentric character including expression arm (16) namely get one's arms around meaning fully understand an issue or situation. (Doctors are having difficulty getting their arms around these new findings) [ODE]. This phraseme is interpreted on the basis of its systematic meanings and a symbolic image of the embracing arms. In a certain communicative situation describing a problematic situation in which a person finds himself rather than a characteristic posture of a person, the meaning shifts. The symbolic image of the embracing arms turns out to be dependent on the context related to an unsolved problem, hopeless position, or other difficulty. Anthropometric character of the image, when a person's nearest and dearest body contributes to the conflict resolution, is extremely positive. Along with the nearness of the human body, the semantic meaning of the word arm contains potential invariant components associated with help or a helper (a hand-a helper, arm (7) used to refer to the holding of a person's arm in support companionship). Combining all this, an individual mentally produces a schematic cognitive image of a person (perhaps himself) embrac- 
ing a problem or a difficulty. It should be noted that this can be an extremely subjective, vivid image or a fuzzy contour corresponding to a certain perceiving or communicating consciousness. Accordingly, while interpreting the situation as a whole, the consciousness infers the meaning of the phraseological unit: "fully understand the nature of the problem/difficulty" [12-14].

The carried out componential analysis of the metaphorical meanings made it possible to determine the frequency of the defined semantic components. Such components as something long/narrow, lateral, projects from a larger structure, and a smaller division of a company/organization indicate that an average native speaker considers an arm as an important lateral part projecting from a larger structure. The performed analysis resulted in objective formulation of an abstract, informative core of the polysemant arm (its lexical invariant): something smaller often long/narrow or lateral that projects from a larger structure/is a division of it.

In view of the fact that the lexeme contains the revealed anthropocentric components connected with a human body capable of generating new associations (based on the LI), it is likely to produce new metaphorical meanings. All the meanings of this word can be called anthropocentric, directly related a person trying to explain the meaning of the objects surrounding him, by means of the structure and function of his body.

Since the anthropocentric reality conceptualization method for characterizing language is both universal and nationally specific, the analyzed lexical invariant of the word arm contains the most significant components of the meaning reflecting such a worldview $[6,7]$.

Thus, metaphorical or invariant development of the semantic structure of this word appears to be a priority.

\section{BIBLIOGRAPHY}

1. Kubryakova E. S. Human factor in language. Language and speech production / E. S. Kubryakova, A. M., Shahnazarovich, L. V. Sakharov; resp. ed. E. S. Kubryakova; USSR Academy of Sciences, Institute of Linguistics. Moscow: Science, 1991. 238 p.

2. Vidmanova E. E., Kulikova N.In. Anthropocentrism as the basis of the semantics of phraseological units (on the example of English idioms). Scientific Notes of Kazan University. Series of Humanitarian Sciences. 2016. Vol. 158. Part 5. Pp. 1308-1314.

3. Vorobieva A. A. Theory of the text: anthropocentric direction / A. A. Vorobiov. M.: Higher School, 2005. 365 p.

4. Yusupova L. G. Imagery in cognitive science. XVII International Scientific and Practical Conference Languages and Culture, Novosibirsk: Center for Development of S Scientific Cooperation, 2015. P. 212.

5. Yusupova L. G. Formation of metaphors. XVII International Scientific and Practical Conference Languages and Culture, Novosibirsk: "Center for Development of Scientific Cooperation", 2015. 212 p.

6. Yusupova L. G., Pesina S. A. Polysemantic word and cognitive memory models. Fundamental scientific researches: theoretical and practical aspects: a collection of materials of the International Scientific and Practical Conference (May 25-26, 2016), Vol. II - Kemerovo: West-Siberian Scientific Center, 2016. 478 p., P. 211-214.

7. Yusupova L. G., Pesina Anthropocentrism as the source of replenishment vocabulary of language. Bulletin of Bashkir University. Publisher: Bashkir state University (Ufa). Vol. 21. No 4. 2016. Pp. 1083-1089.

8. Pesina S., Yusupova L. Words Functioning in Lexicon // Procedia - Social and Behavioral Sciences, Vol. 192. 24 (2015), Pp. 38-43.

9. Pesina S., Latushkina O. Polysemy and Cognition // Procedia - Social and Behavioral Sciences, Vol. 192. 24 (2015). Pp. 486-490.

10. Pesina S., Kostina N. Eidetic analysis in Phenomenology as Try to Solve Polysemy Problem // Procedia - Social and Behavioral Sciences, Vol. 192, 24 (2015). Pp. 33-37.

11. Pesina S., Solonchak T. Lexical Eidos in Linguistics // Procedia - Social and Behavioral Sciences, Vol. 192. 24 (2015). Pp. 764-768.

12. LDCE - Longman Dictionary of Contemporary English. Harlow: Longman Group UK LTD, 1999. 1668 p.

13. MEDAL - Macmillan English Dictionary for Advanced Learners. London: Macmillan Education, 2006. 1652 p.

14. Roget's Thesaurus URL: http://machaut.uchicago.edu/rogets

Поступила в редакциию 23.04.2019 г. 